\title{
Hyperkaliämie: Mit Kammerflimmern in die Notaufnahme
}

\section{Bettina Heberer}

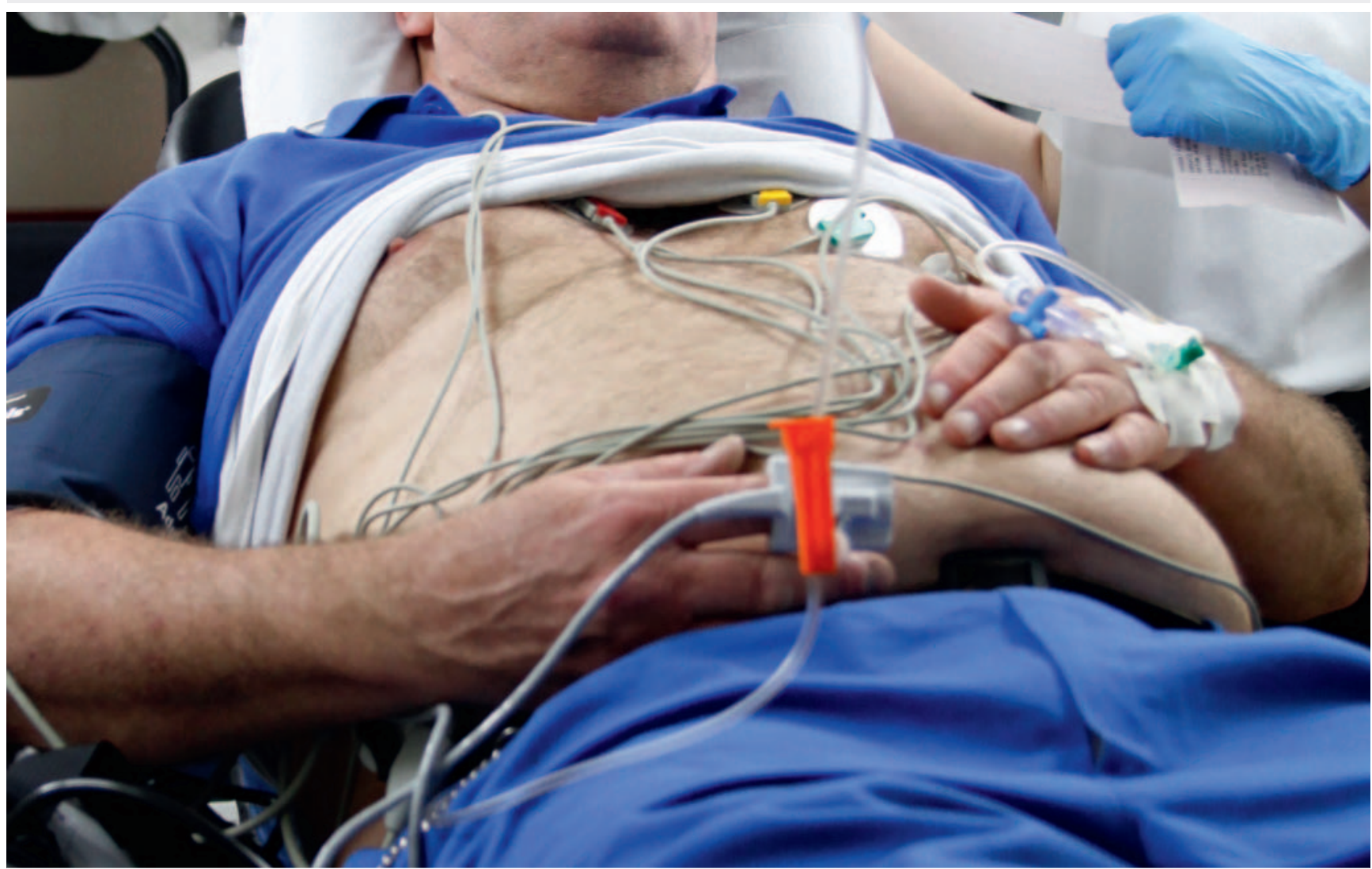

Quelle: Thieme Verlagsgruppe (nachgestellte Situation)

Eine Einsatzmeldung geht über die Leitstelle bei der Rettungswache in Ludwigsburg ein. Ein Patient mit unklaren Symptomen hat den Rettungsdienst gerufen, die Meldung klingt unspektakulär. Beim Eintreffen vor Ort finden die Rettungsassistenten einen sehr schwach wirkenden Patienten vor, der jedoch nicht vital gefährdet wirkt. Auf dem Weg in die Klinik kommt es zum Kammerflimmern.

\section{Die Notfallmeldung}

Es ist kurz vor 4 Uhr morgens während der Nachtschicht, als der damalige Rettungsassistent Rico Kuhnke die Einsatzmeldung entgegennimmt. Gemeinsam mit seinem Kollegen macht er sich auf den Weg. Aufgrund der unspektakulären Einsatzmeldung verzichten sie auf den Einsatz von Martinshorn oder Blaulicht, nehmen jedoch die komplette Ausrüstung mit. „Mein Kollege und ich haben zum Einsatz immer alles mitgenommen, auch wenn es kein Notfalleinsatz war - unsere komplette Ausrüstung mit Notfallkoffer, EKG, Absaugeinheit und Sauerstoff“, erinnert sich Kuhnke.
Nach weniger als 15 Minuten erreichen die beiden Rettungsassistenten die Wohnung des Patienten in einem Stadtteil Ludwigsburgs. Der allein lebende Mann, etwa Mitte 50, öffnet ihnen die Tür. Da er müde und sehr kraftlos wirkt, begleiten Kuhnke und sein Kollege den Patienten ins Schlafzimmer, wo dieser sich gleich wieder ins Bett legt. Die Einsatzmeldung scheint sich jedoch zu bestätigen - es sieht alles nach einem undramatischen Fall aus. Der Patient ist wach und orientiert, als die Rettungsassistenten beginnen, die Anamnese zu erheben. Die Beschwerden sind unspezifisch: Der Patient klagt, ihm sei komisch und es sei ihm den ganzen letzten Tag immer schlechter gegangen. Er fühle sich einfach wahn- 
sinnig erschöpft. Die Frage nach weiteren Symptomen verneint er.

\section{Abklärung der Vitalwerte}

In der Nacht gegen 1 Uhr sei bereits der ärztliche Notdienst da gewesen, so der Patient. Die Nachfrage, was der Arzt gesagt habe, ergibt jedoch keinen besonderen Befund. Die Atmung ist unauffällig, der Puls regelmäßig und gut tastbar. Da der Patient kein Fieber hat, schließen die Rettungsassistenten einen zuerst vermuteten Infekt aus. Auf die Frage nach Vorerkrankungen berichtet der Patient von einer Niereninsuffizienz und regelmäßiger Dialyse. „Da hätten wir vielleicht hellhörig werden müssen“, sagt Kuhnke. „Am nächsten Tag stand die nächste Dialyse an.“

Kuhnke und sein Kollege klären weiter die Vitalwerte ab, messen Blutdruck und Puls, machen eine Blutzuckerkontrolle und zählen die Atmung aus. „Alles, was wir gemessen haben, war bis dahin unauffällig“, erinnert sich Kuhnke, der damals 28 Jahre alt war. Die Kollegen beraten, ob sie den Patienten am nächsten Tag zum Hausarzt schicken sollen, entschließen sich aber, zunächst noch ein EKG zu machen. „Normalerweise würde man nicht bei jedem Patienten sofort ein EKG schreiben. Wir hatten uns jedoch recht lange mit der Anamnese beschäftigt, und der Hintergrund der Symptomatik war völlig unklar“, erinnert er sich. „Das Einzige, was wir noch nicht gemacht hatten, war ein EKG.“

\section{Auffälliger Befund im EKG \\ Das auffällige EKG lässt die Rettungs- assistenten stutzig werden.}

Es zeigt sich zwar ein regelmäßiges EKG mit Sinusrhythmus, jedoch fällt den beiden Rettungsassistenten sofort der wellenförmige QRS-Komplex auf. „Wenn man es kennt, springt einem das sofort ins Auge - ich hatte sowas jedoch noch nie gesehen“, sagt er. Obwohl der Patient nicht vital gefährdet scheint, entschließen sich die Rettungsassistenten, den Patienten in die Klinik zu bringen. „Es kamen 3 Dinge zusammen - das Schwächegefühl als Symptom, die Vorgeschichte mit Niereninsuffizienz und der auffällige EKG-Befund“, sagt Kuhnke. Gegen eine Einweisung ins Krankenhaus erhebt der Patient keine Einwände - er möchte sich einfach wieder besser fühlen. Die beiden Rettungsassistenten tragen den stark geschwächten Patienten im Stuhl in den Rettungswagen.

\section{Transport in die Klinik}

Die Klinik liegt nur etwa 10 Fahrminuten entfernt. Kurz vor dem Erreichen der Notaufnahme wird die Frequenz des EKG immer langsamer. „Die Frequenz war anfangs noch leicht erhöht, da der Patient nervös war. Er wurde dann zunehmend bradykard, damit hatte ich nicht gerechnet. Das hat mir Sorgen gemacht“, sagt Kuhnke. Einen Notarzt nachzurufen, scheint nun keinen Sinn mehr zu machen. Die Klinik ist nur noch 200 Meter entfernt, als es zum Kammerflimmern kommt. „Und plötzlich weiß man dann wieder, was zu tun ist“, sagt Kuhnke und erläutert: „Eigentlich sind die schwierigsten Patienten diejenigen, die keine Vitalbedrohung haben.“

\section{Der Patient wird unter Reanimations- bedingungen in den Schockraum gebracht.}

Kuhnke beginnt, den Patienten mit Herzdruckmassage und Beatmung zu reanimieren, während sein Kollege die Notaufnahme verständigt und den Rettungswagen abstellt, um ihm dann zu Hilfe zu kommen. „Wir haben defibrilliert, und mein Kollege hat weiter beatmet, während ich die Herzdruckmassage gemacht habe“, erzählt Kuhnke. Primär war die Reanimation erfolgreich. „Die Klinik hatte kaum Vorlaufzeit, da wir natürlich keinen Notfall angemeldet hatten“, sagt er. Die Kollegen aus der Notaufnahme kommen zum Fahrzeug und bringen den Patienten in den Schockraum. Nach wenigen Minuten kommt weiteres Klinikpersonal hinzu und löst die beiden Rettungsassistenten ab. Erneut kommt es im Schockraum zu Kammerflimmern.

„Wir haben den Patienten dann zurückgelassen und sind zurück auf die Rettungswache gefahren“, erinnert sich Kuhnke. „Er wurde weiter reanimiert und sofort an eine Hämodialyse angeschlossen. Leider ist er noch in derselben Nacht verstorben“, erzählt Kuhnke.

Während sie in der Klinik sind, wissen die beiden Rettungsassistenten noch nicht, was die Ursache für den Herzstillstand war. „Mein Kollege und ich haben uns danach natürlich in der Klinik informiert und auch eingehend mit der Literatur beschäftigt“, berichtet Kuhnke. Schnell ist der Hintergrund klar - der Patient hatte eine Hyperkaliämie. „Das passt natürlich alles zusammen: Patienten mit Niereninsuffizienz haben gerne mal Elektrolytentgleisungen, insbesondere, wenn sie sich schlecht ernähren oder andere Störfaktoren dazukommen“, weiß er heute. 


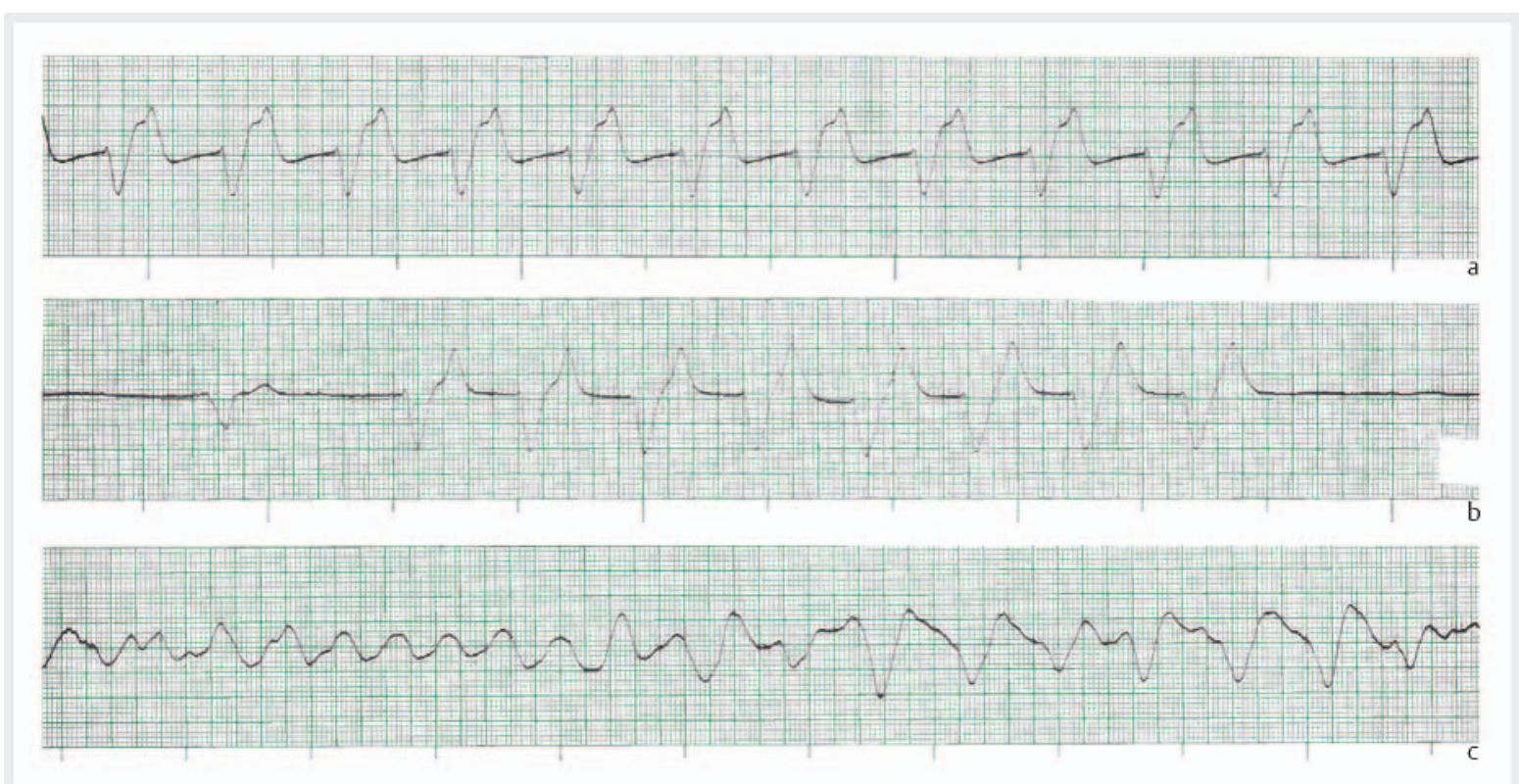

- Abb. 1 EKG des Patienten mit erkennbarer Verschlechterung bis zum Kammerflimmern.

\section{Rückblick}

Kuhnke schaut sehr selbstkritisch auf den damaligen Einsatz zurück - er ist ihm auch nach 20 Jahren noch prägend in Erinnerung geblieben. „Wäre uns klar gewesen, dass es sich um eine Hyperkaliämie handelt, hätten wir frühzeitig großzügig eine Infusionslösung gegeben und erst mal einen Liter infundiert, um einen Verdünnungseffekt des Kaliums zu erreichen“, sagt er und ergänzt: „Sehr viel mehr kann man außerhalb der Klinik auch nicht machen“. Als Rettungsassistent würde er heute bei einem solchen EKG außerdem sofort einen Notarzt nachfordern, jedoch zeitgleich einen schnellen Transport anstreben und nicht auf das Eintreffen des Notarztes warten. „Der Notarzt hätte zwar im konkreten Fall auch nicht mehr machen können, aber zwei Dinge wären anders gelaufen - wir wären zu zweit hinten im Rettungswagen gewesen und hätten mehr Flüssigkeit infundiert“, sagt Kuhnke.

Zum damaligen Zeitpunkt waren die beiden Rettungsassistenten zurückhaltend mit der Infusionsflüssigkeit. „Wir kannten das EKG-Bild nicht und dachten, es muss eine Störung am Herzen sein, und wollten das Herz nicht zu sehr belasten“, erinnert sich Kuhnke. „Wir haben aber bei allen Patienten, die wir damals in die Notaufnahme nach Ludwigsburg gebracht haben, einen venösen Zugang gelegt. Das war der Standard bei uns, auch bei Patienten die, wie in diesem Fall, erst mal wenig kritisch erscheinen“, sagt er.

Der heute 48-Jährige hat nie wieder einen ähnlichen Einsatz erlebt. Mittlerweile ist er seit vielen Jahren in der Lehre tätig, seit 7 Jahren als Gesamtschulleiter der DRK-
Landesschule Baden-Württemberg. „Im Unterricht mögen es die Schüler gar nicht, wenn ich sie mit der Lehre von Elektrolyten traktiere, aber das ist bei mir Standard“, sagt Kuhnke. Auch bei EKG-Interpretationen macht er gerne deutlich, wie wichtig das Wissen um Elektrolyte ist.

\section{Man muss immer achtsam sein und darf die Diagnose nicht zu schnell stellen.}

In den letzten Jahren seien die Einsatzzahlen im Rettungsdienst deutlich angestiegen, jedoch ohne dass es zu einem Anstieg der tatsächlichen Notfälle gekommen sei, so Kuhnke. „Viele Patienten haben unspezifische Symptome, oft ist es nichts Gefährliches, und sie sind meist nicht vital gefährdet." Eine Grundaussage bleibt ihm jedoch aufgrund des erlebten Falls im Kopf: „Auch bei der Vielzahl der Einsätze, die per se eigentlich gar kein Abklären in der Klinik benötigen, die eigentlich auch zum Hausarzt könnten: Man muss immer achtsam sein und darf die Diagnose nicht zu schnell stellen.“

\section{SPANNENDE EINSÄTZE}

Hatten auch Sie einen außergewöhnlichen Einsatz? Ob positiv oder negativ - retten! können Sie davon erzählen und Ihre Kollegen am konkreten Beispiel lernen lassen. Sie erreichen die Redaktion unter 07118931 -905 oder unter retten@thieme.de. 


\section{KOMMENTAR}

von Dr. med. Sönke Müller, hausärztlicher Internist in Neckargemünd, Notarzt im Rettungsdienst und Mitherausgeber von retten!

„Ein Patient mit unklaren Symptomen hat den Rettungsdienst gerufen, die Meldung klingt unspektakulär ...“ Die Zeiten, in denen der Rettungsdienst fast ausschließlich zu (vermeintlich) spektakulären dramatischen Einsätzen ausrücken musste, sind schon lange vorbei: Der Rettungsdienst übernimmt, angesichts des Wandels in der medizinischen Versorgungslandschaft, immer neue, auch unspektakuläre Aufgabenfelder - mit dem Resultat einer Spirale von ständig steigenden Einsatzzahlen und in deren Folge auch ständig steigenden Vorhaltungen an Mensch und Material. Selbst wenn auf den Einsatzmeldern immer noch die Indikationen „V.a. akutes Koronarsyndrom, Dyspnoe, V.a. Apoplex, Sturz aus großer Höhe" u. a. in großer Zahl zu finden sind, wissen doch alle im Rettungsdienst Tätigen aus Erfahrung, dass sich hinter einem Großteil der vermeintlich spektakulären Verdachtsdiagnosen oft nur Harmloses (selbstverständlich nur aus der Sicht des Retters) verbirgt.

Und jetzt kommt sogar noch ein Einsatz, der per se „unspektakulär“ klingt. Was wird das dann erst für ein „Langeweiler“ oder „Ruhestörer“ sein?

Und wirklich: Der Patient schildert unspektakuläre Symptome: „Müdigkeit, Adynamie, und das schon den ganzen Tag, und der ärztliche Bereitschaftsdienst war auch schon da ... Ach ja, und Dialysepatient ist er auch, hat aber morgen schon einen regulären Dialysetermin ...“

Den Patienten zu Hause lassen? Ihn darauf hinweisen, dass es 4 Uhr nachts ist und er doch sicher ab 7.30 Uhr seinen Hausarzt aufsuchen könnte oder dann im Lauf des Tages den Dialysetermin wahrnehmen sollte?
Die Versuchung könnte groß sein - aber nein: Die Professionalität, das Pflichtbewusstsein und die Neugier der Rettungsassistenten sind groß genug, um die Basisdiagnostik mit einem 12-Kanal-EKG abzurunden. Und da fällt dann doch etwas „Komisches“ auf - und ab sofort ist der „UnspektakulärModus" abgeschaltet, der Patient wird konsequent überwacht. Die Dramatik des Kammerflimmerns trifft die Besatzung nicht völlig unvorbereitet! Was lernen wir daraus?

Man kann nicht alles wissen und kennen - die präklinische Diagnose einer Hyperkaliämie kann ohne Weiteres dazu gehören. Aber man sollte niemals darin nachlassen, seine Routine mit den Basisalgorithmen, seine Professionalität mit dem Blick für das „irgendwie Kranksein“ zum Vorteil des Patienten auszuspielen. Auch um 4 Uhr nachts und auch beim „Undramatischen".

\section{Interessenkonflikt}

Die Autoren geben an, dass kein Interessenkonflikt besteht.

\section{Korrespondenzadresse}

\section{Bettina Heberer}

Freie Fachjournalistin

Am Dörrling 3

67273 Herxheim am Berg

Deutschland

Bettina.Heberer@t-online.de

\section{Bibliografie}

DOI https://doi.org/10.1055/a-0629-8760

retten 2019; 8: 221-224

(c) Georg Thieme Verlag KG, Stuttgart · New York ISSN 2193-2387 